\title{
El concepto de proporcionalidad en el contexto del modelo de van Hiele ${ }^{1}$
}

The concept of proportionality in the context of van Hiele model

O conceito de proporcionalidade no contexto do modelo de van Hiele

Recibido: mayo de 2013

Aceptado: agosto de 2013
Tanith Celeny Ibarra Muñoz ${ }^{2}$

Edison Sucerquia Vega ${ }^{3}$

Carlos Mario Jaramillo López ${ }^{4}$

\section{Resumen}

El presente trabajo de investigación aborda los razonamientos que tienen los estudiantes del grado quinto sobre el concepto de proporcionalidad, según el modelo educativo de van Hiele; por lo tanto, el estudio permitió establecer unos descriptores de nivel que caracterizaron y determinaron el nivel de razonamiento en que se encontraban razonando dichos estudiantes. Este estudio se desarrolló en el marco del programa de Maestría en Educación, en la línea de educación matemática, que se desarrolla en una de las sedes regionales de la Universidad de Antioquia, con la participación del grupo de investigación EDUMATH.

Palabras clave: Matemáticas escolares; números; relaciones numéricas; razón y proporcionalidad; segmentos proporcionales; aprendizaje; procesos cognitivos; razonamiento; niveles de razonamiento de van Hiele; entrevista de carácter socrático.

\begin{abstract}
The present research work deals with reasoning with fifth grade students about the concept of proportionality, according to the van Hiele model of education, therefore, the study established level descriptors that characterized and determined the level of reasoning in that these students were reasoning. This study was developed under the Master of Education program in mathematics education line, which takes place in one of the regional headquarters of the University of Antioquia, involving EDUMATH research group.
\end{abstract}

Keywords: school mathematics, numbers, number relationships, reason and proportionality proportional segments; learning cognition, reasoning, reasoning levels of van Hiele; interview Socratic character

\author{
Artículo de Investigación \\ Universidad de Antioquia. Medellin, Colombia. Contacto: tanith927@yahoo.es \\ Universidad de Antioquia. Medellin, Colombia. Contacto: esucerquia@ayura.udea.edu.co \\ Universidad de Antioquia. Medellin, Colombia. Contacto: cama@matematicas.udea.edu.co
}




\section{Resumo}

O presente trabalho de pesquisa lida com raciocínio com alunos do quinto sobre o conceito de proporcionalidade, de acordo com o modelo de van Hiele de educação, os descritores de nível, portanto, o estudo estabeleceu que caracterizaram e determinado o nível de raciocínio que estes alunos estavam raciocínio. Este estudo foi desenvolvido no âmbito do programa de Mestrado em Educação na linha de Educação Matemática, que acontece em uma das sedes regionais da Universidade de Antioquia, envolvendo grupo de pesquisa EDUMATH.

Palavras-chave: matemática da escola, números, relações numéricas, a razão ea proporcionalidade segmentos proporcionais; cognição aprendizagem, raciocínio, raciocínio níveis de van Hiele; entrevista caráter socrático.

\section{Presentación del problema.}

Planteamiento del problema. Los estudiantes del grado quinto de la Institución Educativa Antonio Roldan Betancur, presentan dificultades para comprender el concepto de proporcionalidad; estas dificultades se manifiestan al momento de dar solución a situaciones propias del contexto geométrico que involucran planeamientos de tipo proporcional. Además, no se utiliza un proceso que permita identificar los razonamientos que un estudiante de quinto grado realiza sobre el concepto de proporcionalidad.

Pregunta de investigación. ¿Cómo son los razonamientos que realizan los estudiantes de $5^{\circ}$ de la I. E. Antonio Roldan Betancur, cuando resuelven situaciones de tipo proporcional en contextos geométricos?

Objetivo general. Caracterizar el nivel de razonamiento desde el modelo educativo de van Hiele que realizan los estudiantes de $5^{\circ}$ de la I. E. Antonio Roldan Betancur sobre el concepto de proporcionalidad entre segmentos.

Objetivos específicos. Diseñar e implementar un guión de entrevista de carácter socrático, que permita detectar el nivel de razonamiento en que se encuentra un estudiante sobre el concepto de proporcionalidad, teniendo como componente visual geométrica la división de segmentos.
Establecer descriptores de nivel en el marco del modelo educativo de van Hiele para la comprensión del concepto de proporcionalidad.

\section{Marco referencial.}

Desde los Estándares Básicos de Competencias en Matemáticas (MEN, 2006) el concepto de proporcionalidad es uno de los conceptos centrales tanto desde el pensamiento numérico, como el geométrico y el variacional, destacándose la importancia de que este concepto sea comprendido en niveles de la educación básica, pues es fundamental para otros conceptos.

Definición de los conceptos de razón y proporción. El concepto de razón, desde la revisión realizada en libros de texto de la básica primaria y secundaria, ha sido enseñado solo desde lo numérico como el cociente entre dos cantidades; la expresión aes ab ha sido concebida como a dividido b, lo cual es una manera errónea de entender la razón, pues sólo se hace desde su contexto numérico. Según Freudenthal (1983), desde un contexto fenomenológico, la razón está definida como "una relación de equivalencia en el conjunto de los pares ordenados de números (o valores de magnitud), indicada formalmente por... a:b = c:d, si el par [a,b] es equivalente al par [c, d]" (Freudenthal, 1983, p. 180) 
En esta definición planteada por Freudenthal, la razón no se concibe desde su contexto numérico sino que se fundamenta en sus raíces geométricas, que deben ser el inicio para el trabajo con las razones y las proporciones, como lo plantea Guacaneme (2002) quien afirma que se debe respetar "la naturaleza y esencia de la razón (en tanto relación o pareja) y de la proporción (en tanto equivalencia entre razones o relación entre relaciones), sin que estos conceptos sean contaminados con la idea de número ni de medida" (Guacaneme, 2002, p. 40).

Por lo tanto, en la presente investigación se aborda el concepto de proporcionalidad a partir de las relaciones proporcionales que se establecen entre segmentos; para esto, se retoman las siguientes definiciones propuestas para el concepto de razón entre segmentos y de segmentos proporcionales.

El Modelo Educativo de van Hiele. Es un modelo que fue propuesto por Pierre Marie van Hiele y Dina van Hiele-Geldof, en el año de 1957, inicialmente centró sus aplicaciones en el campo de la geometría, sin embargo, en las últimas décadas ha sido extendido al campo de conceptos del pensamiento matemático avanzado.

El modelo educativo de van Hiele está compuesto por tres aspectos: el insight, los niveles de razonamiento, denominado también aspecto descriptivo del modelo, y las fases de aprendizaje, aspecto prescriptivo.

Tabla 1

\begin{tabular}{|l|l|}
\hline \multicolumn{1}{|c|}{ NIVEL DE RAZONAMIENTO } & \multicolumn{1}{c|}{ DESCRIPCION } \\
\hline Nivel 0, predescritivo & $\begin{array}{l}\text { En este nivel los estudiantes reconocen los } \\
\text { elementos básicos de estudio. }\end{array}$ \\
\hline Nivel 1, de reconocimiento visual & $\begin{array}{l}\text { Los estudiantes ven el objeto de estudio de } \\
\text { manera global, dan características fisicas de } \\
\text { este. }\end{array}$ \\
\hline Nivel 2, de análisis & $\begin{array}{l}\text { En este nivel se identifican y generalizan las } \\
\text { propiedades del concepto estudiado }\end{array}$ \\
\hline Nivel 3, de clasificación o de relaciones & $\begin{array}{l}\text { El estudiante es capaz de establecer } \\
\text { relaciones entre las propiedades del } \\
\text { concepto. }\end{array}$ \\
\hline Nivel 4, de deducción formal & $\begin{array}{l}\text { La característica de este nivel es que se } \\
\text { emplea el razonamiento formal para hacer } \\
\text { demostraciones de las propiedades del } \\
\text { concepto }\end{array}$ \\
\hline
\end{tabular}

Es importante dentro del proceso de investigación determinar y caracterizar los razonamientos que exhiben los estudiantes sobre el concepto objeto de estudio, por lo tanto se hará énfasis en los niveles de razonamiento, que describen con claridad la comprensión que un estudiante tiene ante un determinado concepto, dicho de otra manera, son una estratificación del razonamiento humano. Van Hiele propone 5 niveles de razonamiento que, en el presente proyecto de investigación, serán descritos de acuerdo a Jaramillo \& Esteban (2006):

\section{Metodología}

La investigación, fue abordada desde un enfoque cualitativo a partir de un estudio de casos, ya que era necesario un tratamiento específico que describiera la manera en que los estudiantes realizan los razonamientos $\mathrm{y}$, al mismo tiempo ofrezca información detallada para caracterizar estos razonamientos en relación al concepto de proporcionalidad.

Participantes. Cuatro estudiantes del grado quinto de la Institución Educativa Antonio Roldan Betancur del municipio de Briceño, zona norte del departamento de Antioquia.

Intervención. El principal instrumento que se ha utilizado para la recolección de la información, según la revisión de algunas investigaciones, como la de Jurado \& Londoño (2005) y Santa (2011), las cuales tenían como objetivo caracterizar el nivel de razonamiento que un estudiante presentaba ante un determinado concepto matemático, ha sido la entrevista de carácter socrático. Esta entrevista, es semiestructurada; por lo que se realiza con anterioridad un guion abierto, que se fundamenta en un diálogo debido a su carácter socrático, en el cual las preguntas que se van realizando a los estudiantes tienen un apoyo visual.

El guion de entrevista que se ha diseñado para dar respuesta a la pregunta y los objetivos de investigación, se ha venido refinando gracias a las intervenciones que se realizaron y la revisión cuidadosa de los investigadores.

La realización de la entrevista ha permitido consolidar las preguntas que permitirán que el estudiante exhiba su razonamiento y así lograr determinar y 
caracterizar el nivel de razonamiento en el que se encuentran estos estudiantes con respecto al concepto de proporcionalidad.

La entrevista, no sólo permite determinar el nivel de razonamiento sino que se convierte en una experiencia de aprendizaje, al permitir que el estudiante progrese en los niveles a través de ella. Cada pregunta debe estar diseñada de tal manera que permita la reflexión y la reorganización de las ideas y conocimientos en torno al concepto de proporcionalidad.

\section{Análisis de datos}

El análisis de la información suministrada por los cuatro estudiantes se realizó a través de tres categorías a priori, representación de segmentos, comparando tamaños de segmentos, noción de razón y estableciendo segmentos proporcionales; que van en correspondencia con los niveles propuestos por van Hiele, los razonamientos que evidenciaron los cuatro estudiantes se analizaron por un lado de manera conjunta, para establecer los correspondientes descriptores de nivel y por otro lado de manera individual para determinar el nivel de razonamiento en que se encontraban razonando cada uno de los cuatro estudiantes, para así poder caracterizar el nivel de razonamiento que presentaban los cuatro estudiantes de quinto con respecto al concepto de proporcionalidad entre segmentos.

\section{Conclusiones}

Al abordar la comprensión del concepto de proporcionalidad desde su componente geométrica, se retoman las concepciones que dieron origen a las relaciones proporcionales que en la actualidad hacen parte de nuestra cotidianidad. El estudio de la proporcionalidad, nos ha permitido precisar que el concepto de razón es clave para adquirir la comprensión de éste. Por lo tanto, a partir de los resultados obtenidos, se infiere que la propuesta con la cual se diseñó el guion de entrevista de carácter socrático, permite detectar y caracterizar el nivel de razonamiento en el que se encuentran los estudiantes en el marco del modelo de van Hiele, logrando establecer los descriptores correspondiente para cada nivel de razonamiento.

\section{Referencias}

Freudenthal, H (1983). Didactical Phenomenology of Mathematical Structures. United States of America: EditorialKluwer Academic / Plenum Publishers.

Guacaneme, E. (2002). Una mirada al tratamiento de la proporcionalidad en textos escolares de matemáticas. Revista EMA 2002, 7 (1), 3-42.

Jaramillo, C. \& Esteban, P. (2006). Enseñanza y aprendizaje de las estructuras matemáticas a partir del modelo de Van Hiele. Revista Educación y pedagogía, XVIII (45) (mayoagosto), 109-118.

Jurado, F. \& Londoño, R. (2005). Diseño de una entrevista socrática para la construcción del concepto de suma de una serie vía áreas de figuras planas. Medellín, Colombia: Tesis de Maestría no publicada.

Ministerio de Educación Nacional. (2006). Estándares Básicos de Competencias en Matemáticas. Bogotá, D.C., Colombia: Editorial Magisterio.

Santa, Z. (2011). La elipse como lugar geométrico a través de la geometría del doblado de papel en el contexto de van hiele. Medellín, Colombia: Tesis de Maestría no publicada. 